\title{
The Effect of Amic Ester Precursor on the Water Sorption Behavior and the Stress Relaxation of High Temperature Polyimide Thin Films
}

\author{
Jongchul SeO, Haksoo $\mathrm{HAN}^{\dagger}$, Seungjin KIM,* \\ Hyunsoo ChUnG, and Yung-Il JOE \\ Dept. of Chemical Engineering, Yonsei University, 134 Shinchon-Dong, \\ Sedaemun-Gu, Seoul 120-749, Korea \\ * Saehan Industries Inc. Co., Kiheung-Eup. Yongin-City, \\ Kyunggi-Do 449-900, Korea \\ (Received March 27, 1998)
}

\begin{abstract}
Poly(4,4'-oxydiphenylene pyromellitimide) (PMDA-ODA) and poly(1,4-phenylene pyromellitimide) (PMDA-PDA) polyimides were prepared from poly(amic acid) (PAA) and poly(amic ester) (PAE) precursors, respectively. Water sorption and diffusion behavior in films were measured at $90 \%$ R.H. and $25^{\circ} \mathrm{C}$ using the water sorption method and stress relaxation analysis. Regardless of the method, the diffusion kinetics of water in polyimide thin films was well fitted to Fickian diffusion. For rigid rodlike PMDA-PDA film having high $T_{\mathrm{g}}\left(>400^{\circ} \mathrm{C}\right)$, film prepared from PAE precursor with less chain relaxation and poor stacking efficiency induced higher stress relaxation and diffusion coefficient of water than with the PAA precursor. For semi-flexible PMDA-ODA polyimide film, film prepared from PAE precursor having higher ordered arrangement, higher chain orientation, and well packing structure, had relatively lower diffusion coefficient of water and less stress relaxation than with the PAA precursor. Water diffusion and stress relaxation for the rigid rodlike PMDA-PDA films are relatively slower than for the semi-flexible PMDA-ODA films due to morphological differences.

KEY WORDS Water Sorption / Stress Relaxation / Poly(amic acid) / Poly(amic ester) / Morphology / Polyimide Thin Films /
\end{abstract}

High-temperature polyimides are used for microelectronic devices as interdielectric, alpha-particle-protecting, and passivation layers, owing to relatively high thermal stability, chemical resistance, and mechanical toughness. ${ }^{1}$ However, despite relatively high chemical resistance characteristics, polyimides still sorb water. This water sorption and residual stress of polyimide films induced by sorbing the water molecules cause displacement, crack, delamination, and mechanical failure for the microelectronic devices. ${ }^{2-4}$ Upon uptake of water, dielectric constants and conductivities in polyimide films as well as dielectric loss increase. Consequently, for the development of advanced materials, knowledge of environment effects on the performance of polyimide is essential for the design and selection of structural material.

Polyimides are used in the form of poly(amic acid) (PAA) solution due to the insolubility, high glass transition temperature, and high melting temperature of polyimides. The PAA precursor is very sensitive to the temperature variation and air contact, especially water molecules, resulting in problems of reliability and stability of microelectronic devices. An important improvement over the use of PAA precursor is alkyl ester derivatives from amic side groups. ${ }^{5-7}$ Derivatives, e.g., poly(amic ester) (PAE), have enhanced solubility, longer storage time than the corresponding amic acids, better resistance to hydrolytic degradation, apparent lack of exchange reactions in solution containing different polyimide precursors, and high solids content with low viscosity.

The water sorption behavior was investigated in detail by the water sorption method using an electromicrobalance and the stress relaxation analysis using the Thin Film Stress Measurement System (TFSMS). Water diffusion and stress relaxation kinetics in polyimide thin film were investigated with backbone structure, precursor type, and morphological structure.

\section{EXPERIMENTAL}

\section{Materials and Sample Preparation}

Poly(4,4'-oxydiphenylene pyromellitimide) (PMDAODA) and poly(1,4-diphenylene pyromellitimide) (PMDA-PDA) were used in this study. PAA precursors were prepared in $N$-methyl-2-pyrrolidinone (NMP) through solution polycondensation ${ }^{1-3}$ from PMDA dianhydride and two different diamines: PMDA-PDA and PMDA-ODA PAA precursors. All monomers and solvents were purchased from Aldrich Chemical Co.; pyromellitic dianhydride (PMDA), 1,4-phenylene diamine (PDA), and 4,4'-oxydiphenylene diamine (ODA). The corresponding PAE precursor solutions were prepared by solution polycondensation ${ }^{5-7}$ of diacid chloride and aromatic diamine in NMP; PMDA-PDA and PMDA-ODA PAE precursors. The solid content of polyimide precursors was $15 \mathrm{wt} \%$. The molecular structures of the fully cured polyimides from PAA and PAE precursors are shown in Figure 1.

Polyimide precursors were spin coated on a silicon (100) substrate. The thickness of polyimide thin film was controlled in the range of $12-14$ microns by spinning speed $^{8-12}$ of spin coater. Coated wafers were prebaked at $80^{\circ} \mathrm{C}$ for $30 \mathrm{~min}$ on a hot plate under nitrogen flow. The prebaked samples were then placed in the curing oven and cured under flowing nitrogen by the following cure schedule: ${ }^{8-12} 150^{\circ} \mathrm{C} / 30 \mathrm{~min}, 230^{\circ} \mathrm{C} / 30$ $\min , 300^{\circ} \mathrm{C} / 30 \mathrm{~min}$, and $400^{\circ} \mathrm{C} / 60 \mathrm{~min}$. The ramping rate

\footnotetext{
† To whom correspondence should be addressed (Tel: +82-2-361-2764, Fax: +82-2-312-6401, e-mail: hshan@bubble.yonsei.ac.kr).
} 


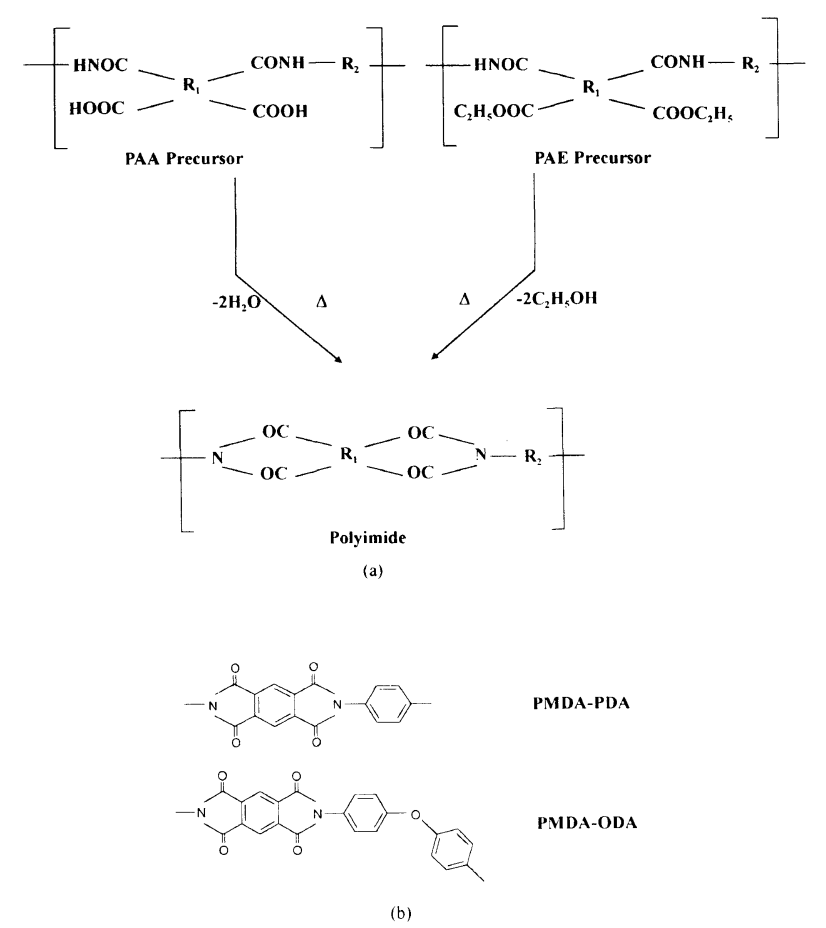

Figure 1. Synthesis and chemical structures of PMDA-PDA and PMDA-ODA polyimide from PAA and PAE precursors: (a) synthetic routes; (b) chemical structures.

for each step was $2.5^{\circ} \mathrm{Cmin}^{-1}$ and cooling rate was $2.0^{\circ} \mathrm{Cmin}^{-1}$. These fully cured films were used to investigate stress relaxation and removed from the substrate after soaking in distilled water for $24 \mathrm{~h}$. The film was washed with distilled water several times, dried, and cut into rectangular pieces approximately $12 \times$ $15 \mathrm{~mm}^{2}$ and fully dried in a vacuum for $24 \mathrm{~h}$ prior to use of water sorption measurement.

\section{Water Sorption and Stress Relaxation Measurement}

Water diffusion kinetics of the polyimide thin films were studied by measurement of mass change as a function of time at $90 \%$ relative humidity (R.H.) and $25^{\circ} \mathrm{C}$, using an electro-microbalance (Cahn Instruments, Model D-200) with a resolution of $0.1 \mu \mathrm{g}$ over $20 \mathrm{mg}$ weight loading. More detail procedures are described in previous studies. $^{8-12}$

All sorption isotherms measured were analyzed with eq 1 below, a typical solution to Fick's second law given by Crank et al. ${ }^{13,14}$ as follows

$$
\frac{M(t)}{M(\infty)}=1-\frac{8}{\pi^{2}} \sum_{m=0}^{\infty} \frac{1}{(2 m+1)^{2}} \exp \left(\frac{-D(2 m+1)^{2} \pi^{2} t}{L^{2}}\right)
$$

$M(t)$ is water uptake at time $t, M(\infty)$ is saturating water uptake at time $t=\infty, D\left(\mathrm{~cm}^{2} \mathrm{~s}^{-1}\right)$ is the diffusion coefficient of water, and $L$, film thickness. The experimental data were plotted with mass uptaking ratio $M(t) / M(\infty)$ as a function of $t^{1 / 2} L^{-1}$. The diffusion coefficient $(D)$ are consequently simulated by fitting eq 1 to normalized experimental data.

Stress relaxation by water sorption was measured as a function of time at $90 \%$ R.H. and $25^{\circ} \mathrm{C}$ using TFSMS. $^{3,15}$ Stress relaxation by sorbing the water

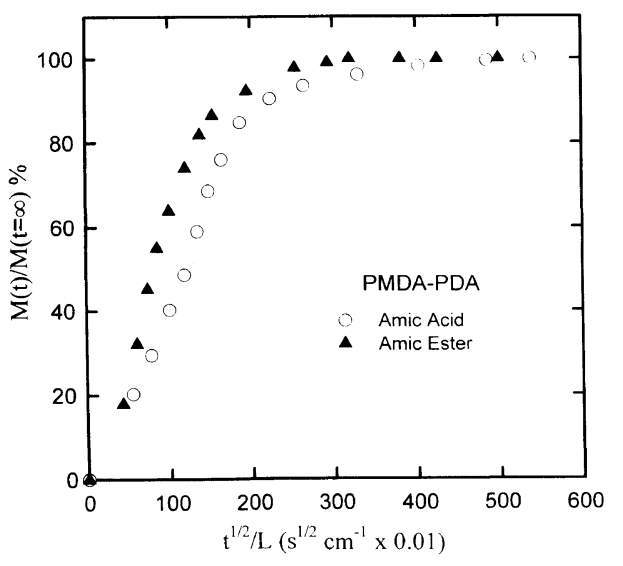

Figure 2. Kinetics of water sorption at $90 \%$ R.H. and $25^{\circ} \mathrm{C}$ for PMDA-PDA polyimides prepared from PAA and PAE precursors.

molecules is represented by the modified Fickian process given by Ree et al. ${ }^{3}$ as follows

$$
\begin{aligned}
\frac{\sigma_{0}-\sigma_{\mathrm{w}}(t)}{\Delta \sigma}= & 1-\frac{8}{\pi^{2}} \sum_{m=1}^{\infty} \frac{1}{(2 m-1)^{2}} \\
& \times \exp \left(-\frac{D(2 m-1)^{2} \pi^{2} t}{4 L^{2}}\right)
\end{aligned}
$$

This equation is applied to a film/substrate structure with constant surface concentration, no concentration gradient at the interface, and initially no water existence in film. $\sigma_{\mathrm{o}}$ is the initial stress at time $t=0, \sigma_{\mathrm{w}}(t)$ is the stress at time $t, \Delta \sigma$ is the difference between stress at $t=0$ and $t=\infty$, and $L$ is thickness of film. The experimental data were plotted with stress relaxation ratio $\left(\sigma_{\mathrm{o}}-\sigma_{\mathrm{w}}(t)\right) / \Delta \sigma$ as a function of $t^{1 / 2} L^{-1}$. The diffusion coefficient using stress relaxation can be simulated by fitting eq 2 to normalized experimental data.

\section{Wide Angle X-Ray Diffraction Measurement}

Wide angle X-ray diffraction (WAXD) measurements were conducted on Rigaku horizontal diffractometer (Model D/Max-200B) with nickel-filtered radiation. The $\mathrm{Cu}-K_{\alpha}$ radiation source $(\lambda=1.54 \AA)$ was operated at $35 \mathrm{kV}$ and $40 \mathrm{~mA}$. Step and scan data were taken in the $\theta / 2 \theta$ mode under computer control at $0.02^{\circ}(2 \theta)$ intervals with a scan speed of $0.1-0.25^{\circ} \mathrm{min}^{-1}$, depending on whether a reflection or transmission scan was being made. Diffraction grams were collected over $3-70^{\circ}(2 \theta)$ with the diffraction vector normal to or in the plane of the film. Stacked film was so arranged that the incident beam would be parallel to the surface for the reflection patterns and perpendicular to the surface for the transmission pattern. The measured X-ray diffraction intensity was corrected for the background run and normalized for film samples by matching the integrated intensity over the range of $68-70^{\circ}(2 \theta)$.

\section{RESULTS AND DISCUSSION}

\section{Rigid Rodlike PMDA-PDA Polyimide}

Rigid rodlike PMDA-PDA and semi-flexible PMDAODA polyimide films were prepared from PAA and PAE precursors, respectively. Using electro-microbalance, 
Table I. Equilibrium water sorptions and diffusion coefficients at $90 \%$ R.H. and $25^{\circ} \mathrm{C}$ for the polyimide thin films

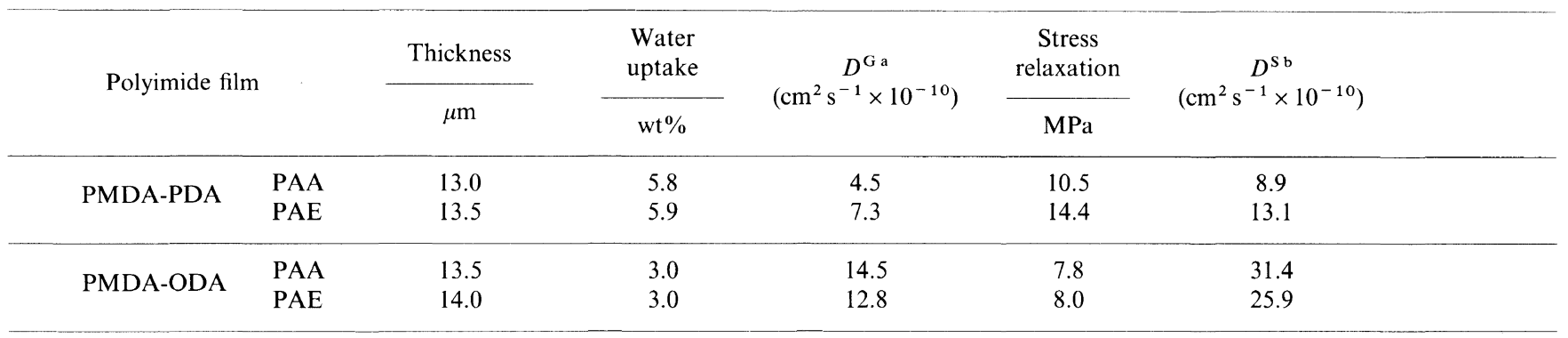

${ }^{a}$ Diffusion coefficient of water at $90 \%$ R.H. and $25^{\circ} \mathrm{C}$ by using the water sorption method. ${ }^{\mathrm{b}}$ Diffusion coefficient of water at $90 \%$ R.H. and $25^{\circ} \mathrm{C}$ by using the stress relaxation method.

water sorption kinetics of fully imidized polyimide thin films was measured at $90 \%$ R.H. and $25^{\circ}$ C. The experimental data were well fitted to eq 2 , which represents the Fickian diffusion. This is consistent with results reported previously. ${ }^{8-12,15-17}$

Typical water sorption isotherms are shown in Figure 2 and analysis results in Table I. As shown in Table I, the diffusion coefficient of water was $4.5 \times 10^{-10} \mathrm{~cm}^{2} \mathrm{~s}^{-1}$ and $7.3 \times 10^{-10} \mathrm{~cm}^{2} \mathrm{~s}^{-1}$ for the PMDA-PDA films prepared from PAA and PAE precursors, respectively. The diffusion coefficient of water in the rigid rodlike PMDA-PDA polyimide film prepared from PAE precursor was significantly larger than for PAA precursor. There was no difference in water uptakes of equilibrium for PMDA-PDA films from prepared PAA and PAE precursors. For fully cured PMDA-PDA polyimide thin films, stress relaxation by sorbing the water molecules was studied at $90 \%$ R.H. and $25^{\circ} \mathrm{C}$, and depicted in Figure 3. Stress relaxation is induced by the creep and moisture uptake, ${ }^{3}$ mainly caused by moisture uptake for polyimide having high glass transition temperature. ${ }^{3}$ For PMDA-PDA polyimide thin film, the diffusion coefficients for stress relaxation by sorbing water were estimated by fitting with eq 3 . The diffusion coefficient of water using stress relaxation were $8.9 \times 10^{-10} \mathrm{~cm}^{2} \mathrm{~s}^{-1}$ for PMDA-PDA polyimide film prepared from PAA precursor and $13.1 \times 10^{-10} \mathrm{~cm}^{2} \mathrm{~s}^{-1}$ for that from PAE precursor as shown in Table I. Regardless of the method, the diffusion coefficient of water for the polyimide film prepared from PAA precursor was smaller than for PAE precursor. However, the diffusion coefficients of polyimide films measured by water sorption method were much smaller than those measured by stress relaxation. Residual stress ${ }^{3}$ generated at the interfaces of films and silicon wafer might cause water diffusion to accelerate into the film as well as interface, leading to the relatively high water diffusion. PMDA-PDA polyimide thin films prepared from PAA and PAE precursors may have the same chemical structures and morphological structures when fully cured. However, due to volatilization of the by-products having different volume during curing process (see Figure 1(a)), morphological structure, ${ }^{1,2,11}$ i.e., orientation, packing, chain relaxation, and crystallinity, can be changed in the fully cured polyimide. Because of the nearness of the curing system to the glass transition temperature, all chain relaxation associated with volatilization may not occur.

To relate structure with sorption behavior of PMDAPDA polyimide thin films, WAXD measurement was

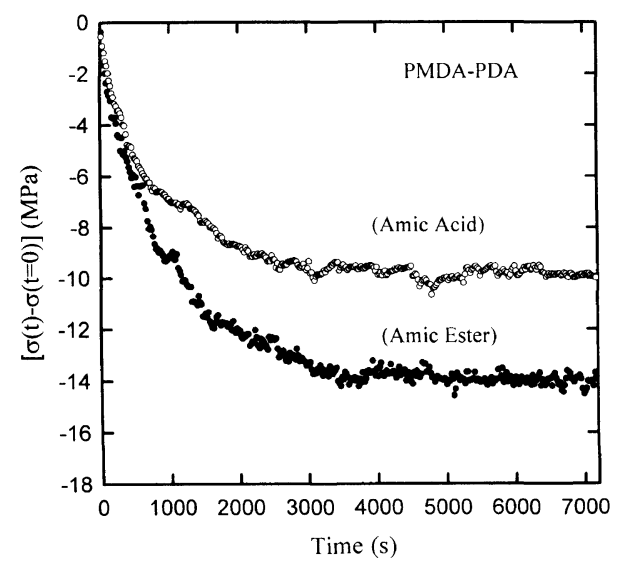

Figure 3. Stress relaxation of PMDA-PDA polyimide films prepared from different precursors on $\mathrm{Si}(100)$ wafers at $90 \%$ R.H. and $25^{\circ} \mathrm{C}$.

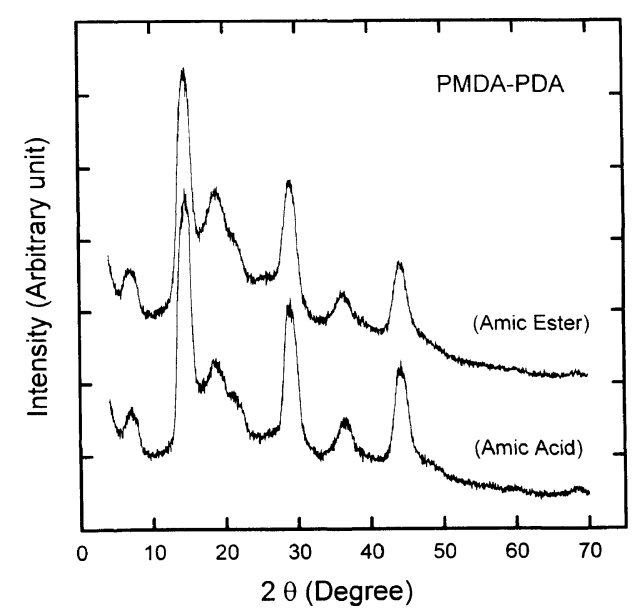

Figure 4. WAXD transmission patterns of PMDA-PDA polyimide films prepared from PAA and PAE precursors.

made for polyimide film prepared from PAA and PAE precursors. The results are presented in Figures 4 and 5. The transmission patterns, which give structural information in the film plane, ${ }^{10,11}$ showed PMDA-PDA polyimide films prepared from PAA and PAE precursors to have multiple diffraction peaks at angles of $2 \theta=7.04$, $14.56,29.12,35.60,44.8^{\circ}$, and one amorphous halo at an angle of $2 \theta=18.80^{\circ}$ as shown in Figure 4. The reflection patterns, which give structural information in the out-of-film plane ${ }^{10,11}$ showed the films to have only one amorphous halo at an angle of $2 \theta=20.8^{\circ}$ as shown in Figure 5. The transmission patterns for PMDA-PDA polyimide films prepared both PAA and PAE precursors 


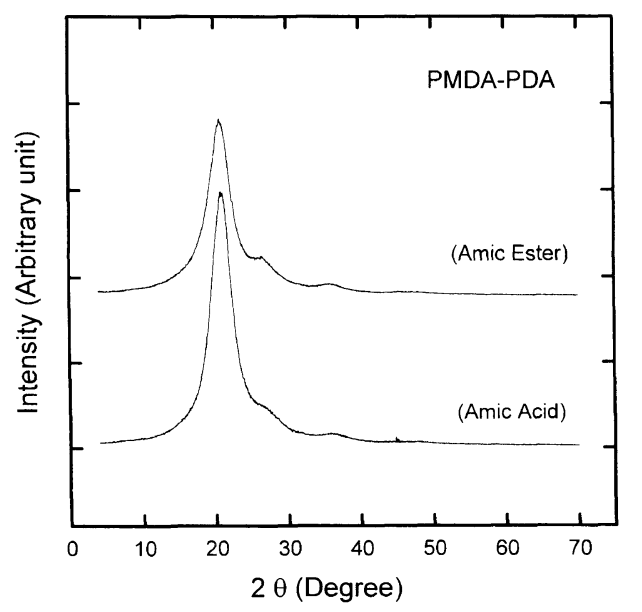

Figure 5. WAXD reflection patterns of PMDA-PDA polyimide films prepared from PAA and PAE precursors.

showed multiple characteristic $(00 l)$ peaks, which indicate a high order and orientation of chain axis direction parallel to the surface of the film. However, the reflection patterns for out-of-plane plane of PMDA-PDA polyimide films showed no characteristic $(00 l)$ peaks but only one amorphous halo. The transmission pattern was not apparently influenced, but the reflection pattern was. As shown in Figure 5, the amorphous halo peak at $20.8^{\circ}$ is relatively much sharper and stronger in intensity for the PMDA-PDA polyimide thin film prepared from the PAA precursor than from the PAE precursor. A comparison of the reflection pattern with the transmission pattern showed not only that PMDA-PDA polyimide film cured on $\mathrm{Si}$ substrate is anisotropic, but also that imide chains are mostly aligned in the film plane. Mean intermolecular distance was estimated from the peak maximum according to the Bragg's equation. ${ }^{16}$ The mean intermolecular distances calculated from the maximum peak of amorphous halos for the transmission were the same value, $4.71 \AA$ for polyimide thin film prepared from PAA and PAE precursors. Mean intermolecular distances calculated from the maximum peak of amorphous halos for reflection were the same value, $4.26 \AA$ for PMDA-PDA polyimide films prepared from PAA and PAE precursors. Mean intermolecular distance calculated from transmission was relatively higher than that from the reflection. This means that polymer chains are more or less well oriented in film plane and well packed in the film thickness direction. We calculated coherence length from broadening $(00 l)$ peaks from Scherrer relationship, ${ }^{16}$ to investigate molecular order and orientation in the chain axis. The coherence lengths of both PMDA-PDA polyimide films prepared from PAE and PAA precursors are the same, $150 \AA$ $\left(2 \theta=7.04^{\circ}\right)$. Hence, having the same values of mean intermolecular distance and coherence length in PMDAPDA polyimide films prepared from PAA and PAE precursors represents no change in chain orientation or molecular ordering during thermal imidization. Therefore, the equilibrium water uptake of rigid rodlike PMDA-PDA polyimide thin films prepared from PAA and PAE precursors was not significantly different as shown in Table I. The diffusion kinetics in the PMDA-PDA polyimide films prepared from PAA and

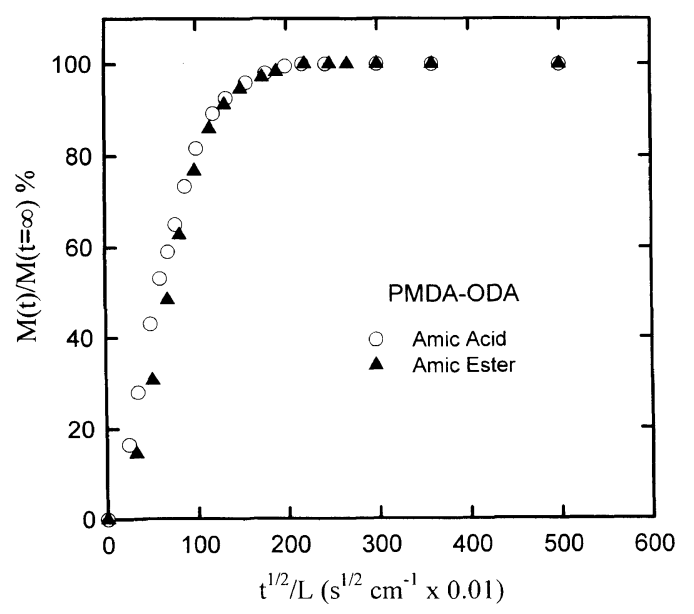

Figure 6. Kinetics of water sorption at $90 \%$ R.H. and $25^{\circ} \mathrm{C}$ for PMDA-ODA polyimides prepared from PAA and PAE precursors.

PAE precursors were dramatically different. $T_{\mathrm{g}}$ of PMDA-PDA film cannot be measured and may be above $500^{\circ} \mathrm{C}^{11,17,18}$ During curing, the rigid PMDA-PDA polymer chain may be immediately frozen because of the higher $T_{\mathrm{g}}$ than the final temperature of curing. During curing of polyimides prepared from PAA and PAE precursors, by-products of two water molecules for PAA and more or less larger by-products for PAE per repeating unit are released from chain units. Volatilization of by-products may induce less chain relaxation and poor stacking efficiency in the fully cured rigid rodlike PMDA-PDA polyimide prepared from PAE precursor than that from PAA precursor. Hence, for a rigid rodlike PMDA-PDA polyimide having high $T_{\mathrm{g}}\left(>400^{\circ} \mathrm{C}\right)$, polyimide prepared from PAE precursor with less degree of chain relaxation may induce higher stress relaxation and higher stress relaxation rate as shown in Figure 3 and Table I. This is in good agreement with the diffusion coefficient by water sorption as shown in Figure 2 .

\section{Semi-Flexible PMDA-ODA Polyimide}

The diffusion of semi-flexible PMDA-ODA polyimides prepared from PAA and PAE precursors are dramatically different from those of the rigid rodlike PMDA-PDA polyimides prepared from PAA and PAE precursors. As shown in Table I, the diffusion coefficient of water for the semi-flexible structure PMDA-ODA film prepared from PAA precursor was $14.5 \times 10^{-10} \mathrm{~cm}^{2} \mathrm{~s}^{-1}$. The diffusion coefficient of water for PMDA-ODA film prepared from PAE precursor was $12.8 \times 10^{-10} \mathrm{~cm}^{2} \mathrm{~s}^{-1}$. Unlike PMDA-PDA polyimide, the diffusion coefficient of water in PMDA-ODA polyimide films prepared from PAA precursor was more or less larger than that from PAE precursor as shown in Figure 6. There was no difference in equilibrium water uptake for the PMDAODA films regardless of the precursor. Stress relaxation by sorbing the water molecules was studied at $90 \%$ R.H. and $25^{\circ} \mathrm{C}$ for the semi-flexible PMDA-ODA polyimide (see Figure 7). The diffusion coefficient of water using stress relaxation was $31.4 \times 10^{-10} \mathrm{~cm}^{2} \mathrm{~s}^{-1}$ for the PMDA-ODA polyimide film prepared from PAA precursor and $25.9 \times 10^{-10} \mathrm{~cm}^{2} \mathrm{~s}^{-1}$ for that from PAE precursor as shown in Table I. In comparison of the measuring methods, the diffusion coefficients of water in 


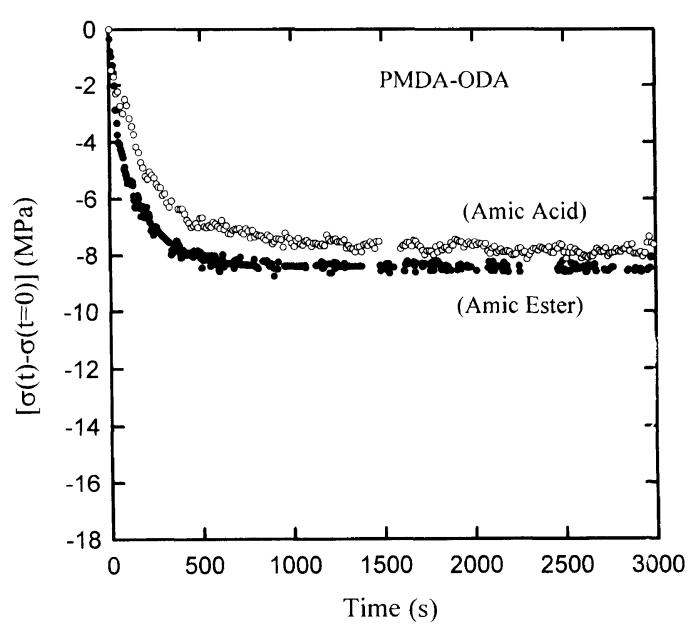

Figure 7. Stress relaxation of PMDA-ODA polyimide films prepared from different precursors on $\mathrm{Si}(100)$ wafers at $90 \%$ R.H. and $25^{\circ} \mathrm{C}$.

polyimide thin films by water sorption method were smaller than those by stress relaxation. This is in good agreement with PMDA-PDA polyimide. However, the diffusion coefficient of water for the semi-flexible PMDA-ODA polyimide prepared from PAE precursor was more or less lower than that from PAA precursor regardless of the measuring method. Unlike the rigid rodlike PMDA-PDA polyimide, relatively larger amic ester group in PAE precursor did not show a significant effect on water sorption kinetics of the semi-flexible PMDA-ODA polyimide. There thus may be no big difference in morphological structure, which changes water sorption kinetics.

WAXD measurement was performed for PMDAODA polyimide films prepared from PAA and PAE precursors. The results are presented in Figures 8 and 9. The transmission patterns showed PMDA-ODA polyimide films prepared from PAA and PAE precursors to have one sharp diffraction peak at a low angle of $2 \theta=5.74^{\circ}$ and one amorphous halo at an angle of $2 \theta=18.2^{\circ}$ as shown in Figure 8. The reflection patterns showed PMDA-ODA polyimide films prepared from PAA and PAE precursors to have no characteristic peaks and only one diffuse and amorphous halo at an angle of $2 \theta=18.5^{\circ}$ as shown in Figure 9. As with the rigid rodlike PMDA-PDA polyimide films, the transmission patterns for PMDA-ODA polyimide films showed the characteristic $(002)$ peak $\left(2 \theta=5.74^{\circ}\right)$ indicating high order and orientation of the chain axis direction parallel to the surface of the film. The (002) diffraction peak of film prepared from PAE precursor was higher than from PAA precursor for the transmission patterns. A comparison of the intensity indicates that PMDA-ODA polyimide prepared from PAE precursor is more ordered in the $c$-axis direction than from the PAA precursor. Hinged type PMDA-ODA films ${ }^{1}$ are inherently more flexible and larger volume shrinkage with ethanol molecules makes possible a more ordered arrangement of the zigzag in the $c$-axis direction. In reflection patterns, though there were no characteristic diffraction peaks, the PMDA-ODA polyimide film prepared from PAE precursor appeared to have a slight peak at about $2 \theta=25^{\circ}$. The PMDA-ODA polyimide film prepared from PAA precursor shows no significant order while that from PAE precursor does.

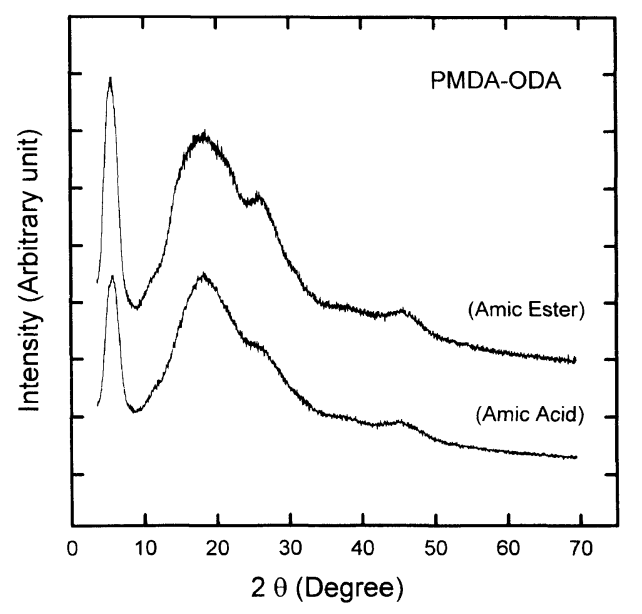

Figure 8. WAXD transmission patterns of PMDA-ODA polyimide films prepared from PAA and PAE precursors.

For transmission patterns, mean intermolecular distances using the Bragg's equation ${ }^{16}$ are $4.87 \AA$ and $4.79 \AA$ for the PMDA-ODA polyimide films prepared from PAA and PAE precursors, respectively. For reflection patterns, mean intermolecular distances ${ }^{16}$ are $4.81 \AA$ and $4.65 \AA$ for the PMDA-ODA polyimide films prepared from PAA and PAE precursors, respectively. For semi-flexible PMDA-ODA polyimide films, coherence lengths are $86 \AA$ and $90 \AA$ for PMDA-ODA polyimide films prepared from PAA and PAE precursors, respectively. Thus, unlike rigid rodlike PMDA-PDA polyimide thin film, the semi-flexible PMDA-ODA polyimide film prepared from PAE precursor has more or less higher ordered arrangement and chain orientation, and is more well packed than that from PAA. For these reasons, water diffusion rate was relatively lower for the PMDA-ODA polyimide prepared from PAE precursor than that from PAA precursor. These are consistent with stress relaxation rates as shown in Table I and Figure 7.

PMDA-ODA polyimides are relatively more flexible and have lower $T_{\mathrm{g}}$ than PMDA-PDA polyimides. $T_{\mathrm{g}}$ of PMDA-ODA polyimide was found to be $380^{\circ} \mathrm{C}$ below the final curing temperature. ${ }^{8,11,18}$ During curing, two water molecules for PAA and relatively larger byproducts for PAE per repeating unit are released from chain units (see Figure 1(a)), and this may change chain relaxation and chain stacking efficiency of final products. Due to the lower $T_{\mathrm{g}}$ than final curing temperature, semi-flexible PMDA-ODA polyimides prepared from the PAA and PAE precursors have enough chain mobility to rearrange the chain. As described previously, ${ }^{11}$ the storage modulus of PMDA-ODA decreased dramatically near $T_{\mathrm{g}}$, which means increase of chain mobility. This increased chain mobility may induce larger chain relaxation for semi-flexible PMDA-ODA polyimide. Due to the relatively lower $T_{\mathrm{g}}$ and increased chain mobility, PMDA-ODA polyimides prepared from PAA and PAE precursors have enough chain relaxation. Therefore, there might be no significant differences in stress relaxation of PMDA-ODA polyimide thin films prepared from PAA and PAE precursors. This shows a good agreement with water sorption results in Table I and Figure 6. 


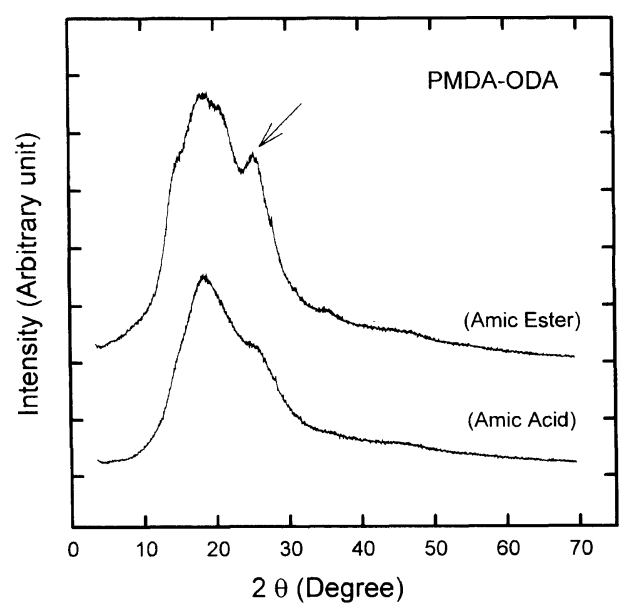

Figure 9. WAXD reflection patterns of PMDA-ODA polyimide films prepared from PAA and PAE precursors.

\section{Effects of Backbone Structure}

As shown in Figures 2, 3, 6, 7, and Table I, for the diffusion coefficient by using water sorption and stress relaxation, the rigid rodlike PMDA-PDA polyimide has relatively lower values than the semi-flexible PMDAODA polyimide. Rigid rodlike PMDA-PDA polyimides have larger water uptake and stress relaxation than semi-flexible PMDA-ODA polyimide. PMDA-PDA and PMDA-ODA polyimides in films consist of ordered and disordered phases. ${ }^{8,10,11,19,20}$ The ordered phases are impenetrable obstacles which diminish volume available for transport. When parts of the ordered phase become high enough, they may constrain relaxation in the amorphous phase and reduce the ability of molecules to squeeze through. ${ }^{8}$ From WAXD transmission and reflection patterns (see Figures 4, 5, 8, and 9) of PMDA-PDA and PMDA-ODA, it may be deduced that PMDA-PDA polyimides are relatively more ordered along the chain axis and more oriented in the film plane direction than PMDA-ODA polyimides. The rates of water diffusion and stress relaxation are thus higher for semi-flexible PMDA-ODA films than the rigid rodlike PMDA-PDA films. However, PMDA-PDA exhibited relatively higher water uptake and stress relaxation than PMDA-ODA. PMDA-PDA having $T_{\mathrm{g}}$ above the final curing temperature may thus induce lower stacking efficiency and less chain relaxation than PMDA-ODA. That is, PMDA-PDA has $T_{\mathrm{g}}$ above the final temperature of curing, and polymer chains may be immediately frozen because of higher glass transition temperature over the final temperature of curing process. In final stages of curing, volume relaxation and chain mobility slow down, and there are very limited mobility and less chain relaxation to permit chain organization to increase packing efficiency. Therefore, PMDAPDA may have larger water sorption and higher stress relaxation as shown in Table $\mathrm{I}$.

\section{CONCLUSIONS}

Rigid rodlike PMDA-PDA and semi-flexible PMDAODA polyimide films thermally imidized from the PAA and PAE precursor solutions were investigated. In spite of differences in measuring method, water diffusion kinetics of the rigid rodlike PMDA-PDA and the semi-flexible PMDA-ODA polyimide thin films in this study was well fitted to Fickian diffusion model.

For rigid rodlike PMDA-PDA polyimide having high $T_{\mathrm{g}}\left(>400^{\circ} \mathrm{C}\right)$, PMDA-PDA polyimide prepared from PAE precursor shows less chain relaxation and poor stacking efficiency than from the PAA precursor. For the rigid rodlike PMDA-PDA polyimide prepared from PAE precursor, volatilization of larger molecules hinders enough chain relaxation, resulting in lower stacking efficiency during curing. This may induce higher diffusion coefficient of water and stress relaxation in PMDA-PDA polyimide prepared from PAE precursor than that from PAA precursor.

For the semi-flexible PMDA-ODA polyimide having relatively lower $T_{\mathrm{g}}\left(<400^{\circ} \mathrm{C}\right)$, more ordered structure, higher chain orientation, and well packing structure resulted in the polyimide prepared from the large molecule PAE precursor. Therefore, the PMDA-ODA polyimide prepared from PAE precursor shows lower diffusion coefficient of water than from the PAA precursor. However, these morphological changes had no significant effect on stress relaxation or equilibrium water uptake.

The rigid rodlike PMDA-PDA polyimide having high $T_{\mathrm{g}}\left(>400^{\circ} \mathrm{C}\right)$ over its final curing temperature shows lower stacking efficiency and less chain relaxation than the semi-flexible PMDA-ODA polyimide having low $T_{\mathrm{g}}$ $\left(<400^{\circ} \mathrm{C}\right)$. Therefore, the rigid rodlike PMDA-PDA polyimide shows larger water sorption and higher stress relaxation than the semi-flexible PMDA-ODA polyimide.

Acknowledgments. We thank IITA (Institute of Information Technology Assessment, U96-141) for financial support of this work.

\section{REFERENCES}

1. M. I. Bessonov and V. A. Zubkov, "Polyamic Acids and Polyimides; Synthesis, Transformations, and Structure," CRC Press, Boca Raton, FL, 1993.

2. M. K. Ghosh and K. L. Mittal, "Polyimides; Fundamentals and Applications," Marcel Dekker, Inc., New York, N.Y., 1996.

3. C. Feger, M. M. Khojasteh, and M. S. Htoo, "Advances in Polyimide Science and Technology," TECHNOMIC, Lancaster, PA, 1993.

4. L. F. Thompson, C. G. Wilson, and S. Tagawa, "Polymers for Microelectronics; Resist and Dielectrics," ACS Symposium Series 537, 1992.

5. K. H. Becker and H. W. Schmidt, Macromolecules, 25, 6784 (1992).

6. Y.-T. Chern, Macromol. Chem. Phys., 196, 3217 (1995).

7. F. M. Houlihan, B. J. Bachman, C. W. Wilins, and C. A. Pryde, Polym. Mater. Sci. Eng., 59, 225 (1998).

8. M. Ree, H. Han, and C. C. Gryte, High Perform. Polym., 6, 321 (1994).

9. M. Ree, S. Swanson, and W. Volksen, Polymer, 34, 1423 (1993).

10. H. Han, C. C. Gryte, and M. Ree, Polymer, 36, 1663 (1995).

11. H. Han, J. Seo, M. Ree, S. M. Pyo, and C. C. Gryte, Polymer, 39, 2963 (1998)

12. M. Ree, H. Han, and C. C. Gryte, J. Polym. Sci., Polym. Phys., 33, 505 (1995).

13. J. Crank, "The Mathematics of Diffusion," Clarendon Press, Oxford, 1976

14. J. Crank and G. S. Park, "Diffusion in Polymers," Academic Press, London, 1968. 
15. J. H. Jou, R. Huang, P. T. Huang, and W. P. Shen, J. Appl. Polym. Sci., 43, 857 (1991).

16. B. D. Cullity, "Elements of X-Ray Diffraction," 2nd ed, Addison-Wesley Publishing Company, Inc., London, 1977.

17. M. Ree, K. Kim, S. H. Woo, and H. Chang, J. Appl. Phys., 81, 698 (1997).
18. W. Huang, Y. Tong, J. Xu, and M. Dong, J. Polym. Sci., Polym. Chem., 35, 143 (1997).

19. T. P. Russell, J. Polym. Phys., 22, 1105 (1994).

20. J. G. Van Alsten and J. C. Coburn, Macromolecules, 27, 3746 (1994). 